\title{
Structure of Cholesterol/Lipid Ordered Domains in Monolayers and Single Hydrated Bilayers**
}

\author{
Roy Ziblat, Kristian Kjaer, Leslie Leiserowitz,* and Lia Addadi*
}

Cell membranes are currently thought to consist of different lipid phases and domains at the nanometer scale. ${ }^{[1]}$ These domains (also known as "lipid rafts") differ in composition, structure, and stability and consequently in properties and function. They selectively incorporate or exclude specific proteins, and thereby fulfill an important function in cell activity and signaling. ${ }^{[2]}$

The compositions of the different membranes in the cell organelles differ greatly according to their function. ${ }^{[3]} \mathrm{Mem}-$ brane compositions also vary substantially among different types of cells and among organelles within the same cell. To date, thousands of different lipid molecules have been found to exist in the cell membranes, often differing one from the other only in chain length or other parameters not associated with functional-group interaction. ${ }^{[4]}$ The possible advantage of this multiplicity and redundancy may be explained at least in part by the different interactions between the lipid chains, which in turn regulate the composition, organization, and functional parameters of the membrane. Understanding the rules that govern membrane structure is thus crucial to understanding cell biology.

Grazing-incidence X-ray diffraction $(\mathrm{GIXD})^{[5]}$ can provide direct information on the organization of laterally

[*] Prof. L. Leiserowitz

Department of Materials and Interfaces

Weizmann Institute of Science, 76100 Rehovot (Israel)

Fax: $(+972)$ 89-344-138

E-mail: leslie.leiserowitz@weizmann.ac.il

R. Ziblat, Prof. L. Addadi

Department of Structural Biology

Weizmann Institute of Science, 76100 Rehovot (Israel)

Fax: $(+972)$ 89-344-136

E-mail: lia.addadi@weizmann.ac.il

Homepage: http://www.weizmann.ac.il/sb/faculty_pages/Addadi/ home.html

Dr. K. Kjaer

Max Planck Institute of Colloids and Interfaces

14476 Potsdam-Golm (Germany)

and

Niels Bohr Institute, University of Copenhagen

Copenhagen (Denmark)

[***] We thank HASYLAB for synchrotron beamtime. This work was supported by the Israel Science Foundation and the Clore Center for Biological Physics. We are grateful for the support of the Helen and Martin Kimmel Center for Nanoscale Science. We thank Dr. Matthias A. Fenner from Agilent Technologies for his help with characterization of the bilayer samples by AFM. L.A. is the incumbent of the Dorothy and Patrick Gorman Professorial Chair of Biological Ultrastructure.

Dupporting information for this article is available on the WWW under http://dx.doi.org/10.1002/anie.200903847. periodically ordered lipid sheets. GIXD has been used for studying the structure of two-dimensional (2D) lipid monolayers at the air/water interface. ${ }^{[6]}$ Membranes are, however, bilayers composed of two opposing juxtaposed monolayers, which interact with each other at the hydrophobic side. The structure of the bilayer may thus be conceivably different from that of the corresponding monolayer. ${ }^{[6 b, d]}$ To guarantee preservation of the structural integrity of a membrane bilayer with hydrophobic interior and hydrophilic external surfaces, wetting on both sides of the bilayer is required. However, because of the strong X-ray background scattering contribution of liquid water, until now GIXD experiments have been reported on lipid films that were dry at the side of the impinging X-ray beam, ${ }^{[6,7]}$ or on stacks of bilayers. ${ }^{[8]}$ Such studies should be compared with those of individual hydrated lipid bilayers.

This was recently made possible by working with highenergy beams, for which the attenuation resulting from photoelectric absorption by water is weaker, thus preserving more of the X-ray intensity even when the sample is immersed in water. Such a pioneering experiment was performed recently by Miller et al., who obtained a diffraction signal from a single 1,2-dipalmitoyl-sn-glycero-3-phosphoethanolamine (DPPE) bilayer. ${ }^{[9]}$ The bilayer was directly deposited on a silicon wafer, thus limiting the diffusion kinetics of the molecules in contact with the wafer. The intensity of the signal was strongly attenuated by the presence of water, which, additionally, gave rise to strong background scattering. Therefore, this method might not succeed in measuring samples with a low amount of crystalline bilayer material, as in this study.

Herein, we report the crystal structure of domains formed in bilayers that are composed of sphingomyelin (SM) and cholesterol (Chol), considered to be the main components of ordered lipid domains in cells. ${ }^{[2]}$ Structure measurements were derived by applying a new humidity control method, which we introduce here. The principle is to work close to the dew point of water and adjust the thickness of the condensed water layer on top of the sample through controlling the temperature differences between the sample and the humidified gas (Figure 1).

The lipid bilayer sample is placed in a humidity chamber and covered with a thick layer of water. Evaporation is then initiated by increasing the temperature of the sample to $20^{\circ} \mathrm{C}$, thereby reducing the relative humidity $(\mathrm{RH})$ above the bilayer to $42 \%$ (Figure 1). The RH is then increased gradually as the water layer thins, by cooling the sample to $6.7^{\circ} \mathrm{C}$. Under these conditions, the RH above the sample is $95.7 \%$ and is thereafter kept rigorously constant. Bilayers are deposited by adapting Langmuir-Blodgett/Langmuir-Schaeffer techni- 
a)

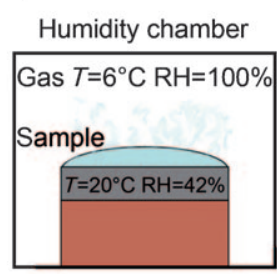

b)

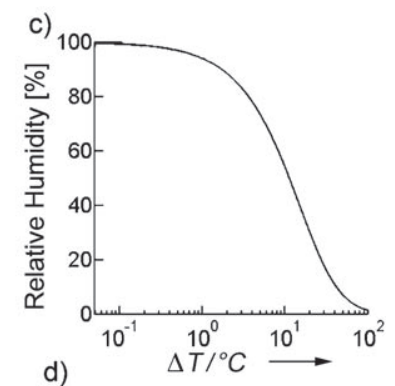

d)
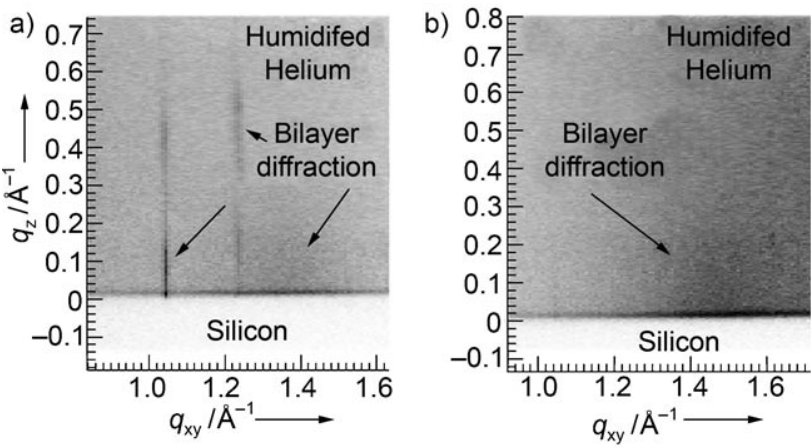

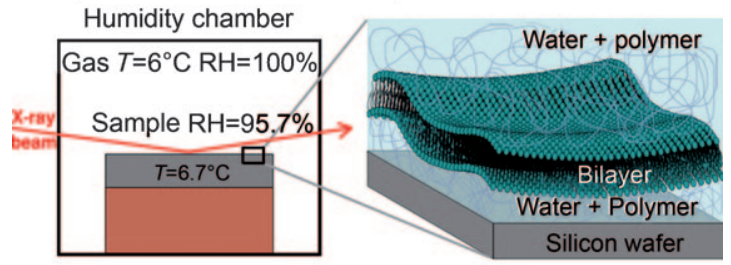

Figure 1. a) Experimental setup: The supported bilayer covered with a thick layer of water is placed inside the chamber, and the water layer is evaporated. b) GIXD is observed when the water layer is less than $1 \mu \mathrm{m}$ in thickness. c) Plot of the $\mathrm{RH}$ above the sample as a function of its temperature difference from that of the surrounding gas. The gas is kept at $6^{\circ} \mathrm{C}$ and $\mathrm{RH}=100 \%$. d) The polymer-supported bilayer.

ques, and are sandwiched between two thin layers of polyethyleneimine. The polymer serves as a water reservoir that prevents dehydration of the sample while affording the bilayer free diffusion, thus better reproducing natural conditions. ${ }^{[10]}$ For a detailed description of sample preparation and characterization by atomic force microscopy (AFM), see the Supporting Information. AFM studies (data not shown) show that in lipid bilayers that are not fully hydrated, the bilayer structure is irreparably damaged.

Figure 2 shows the GIXD pattern obtained from SM, Chol, and 1-palmitoyl-2-oleoyl-sn-glycero-3-phosphocholine (POPC) bilayers using the humidity chamber, and the GIXD pattern from a monolayer of the same composition, obtained using a liquid surface diffractometer. Since POPC is unsaturated it does not phase mix with SM and Chol. It does, however, maintain the lipid bilayer intact for longer periods. The images in Figure 2 were derived directly from raw data and were not processed in any way. The diffraction data are represented as Bragg peaks $I\left(q_{x y}\right)$, the integral of the GIXD pattern $I\left(q_{x y} q_{z}\right)$ over $q_{z}$, and Bragg rod intensity profiles $I\left(q_{z}\right)$, the scattered intensity $I\left(q_{x y}, q_{z}\right)$ integrated across the $q_{x y}$ range of each Bragg peak (Figure 3). The $q_{x y}$ positions of the Bragg peaks yield the lattice repeat distances $d=2 \pi / q_{x y}$, which are indexed by Miller indices $h, k$ to yield the unit cell dimensions. The full width at half maximum (FWHM) of the Bragg peaks yields the $2 \mathrm{D}$ crystalline coherence length $L_{x y} \approx 0.9(2 \pi) /$ $\operatorname{FWHM}\left(q_{x y}\right)$. The Bragg rod profile along $q_{z}$ similarly gives a measure of the thickness of the crystalline film. ${ }^{[\mathrm{a}]}$

Figure 3 compares the Bragg peaks and rods from bilayers composed of SM:Chol:POPC $=54: 36: 10$ and 36:54:10 $\mathrm{mol} \%$, respectively, and a monolayer with a composition ratio of $\mathrm{SM}: \mathrm{Chol}: \mathrm{POPC}=36: 54: 10 \mathrm{~mol} \%$. The monolayer shows one broad peak at $q_{x y}=1.24 \AA^{-1}$ with a coherence length of $2.3 \mathrm{~nm}$, which belongs to a SM:Chol mixed phase. ${ }^{[6,11]}$ By

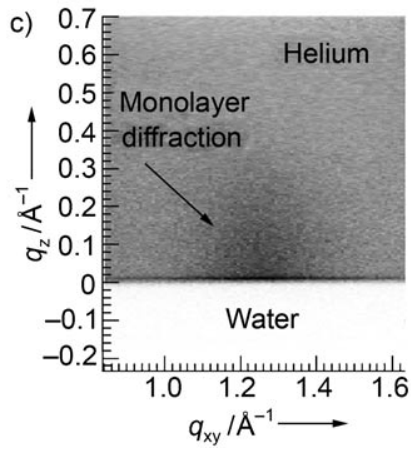

Figure 2. Diffracted intensity signals from $a, b)$ single hydrated bilayers on Si wafers with compositions SM:Chol:POPC $=36: 54: 10$ (a) and $54: 36: 10 \mathrm{~mol} \%$ (b); and c) the monolayer at the water/He interface with composition SM:Chol:POPC $=36: 54: 10 \mathrm{~mol} \%$. The images were produced directly from the raw data and were not modified in any way. Black represents the highest intensity.

comparison, the bilayer with the same composition shows two crystalline phases: a broad Bragg peak located at $q_{x y}=$ $1.35 \AA^{-1}$ with a coherence length of $1.7 \mathrm{~nm}$ that belongs to a SM:Chol mixed phase, and two sharp peaks located at $q_{x y}=$ $1.04,1.23 \AA^{-1}$ that belong to a highly crystalline pure Chol phase with a high coherence length of $(40 \pm 10) \mathrm{nm}$. The latter corresponds to a well-known and characterized bilayer structure, which is the basic motif of the macroscopically metastable phase of Chol monohydrate. ${ }^{[6 \mathrm{~d}]}$ The Bragg rods of these peaks, which are evidence of the bilayer motif, yield a thickness of $(35 \pm 5) \AA$ (Figure $3 \mathrm{~b}$ and $\mathrm{c}$ ). The Bragg rods of the mixed SM:Chol phase, however, show a thickness of $(14 \pm 3) \AA$, which corresponds to the major part of the alkyl chain (Figure $3 \mathrm{~d}$ ), thus indicating that each leaflet of this bilayer phase diffracts independently (Figure 4). Had the two SM:Chol leaflets been arranged in crystalline registry, the Bragg rod would have indicated a film thickness of at least $26 \AA$. The Bragg rods also show that the bilayer sample is flat, as seen from the similarity of their shape along $q_{z}$ to that of the flat monolayer (Figure $3 \mathrm{~d}$ ). Had the bilayer been curved, the shape of the Bragg rods would have been significantly different.

At the ratio SM:Chol:POPC $=54: 36: 10 \mathrm{~mol} \%$ we observe a broad Bragg peak at $q_{x y}=1.47 \AA^{-1}$ with a coherence length of $1.9 \mathrm{~nm}$, which belongs to the SM:Chol mixed phase with a corresponding Bragg rod yielding monolayer thickness, and weak peaks located at $q_{x y}=1.04,1.23 \AA^{-1}$ belonging to the pure Chol crystalline bilayer motif. The intensities of 

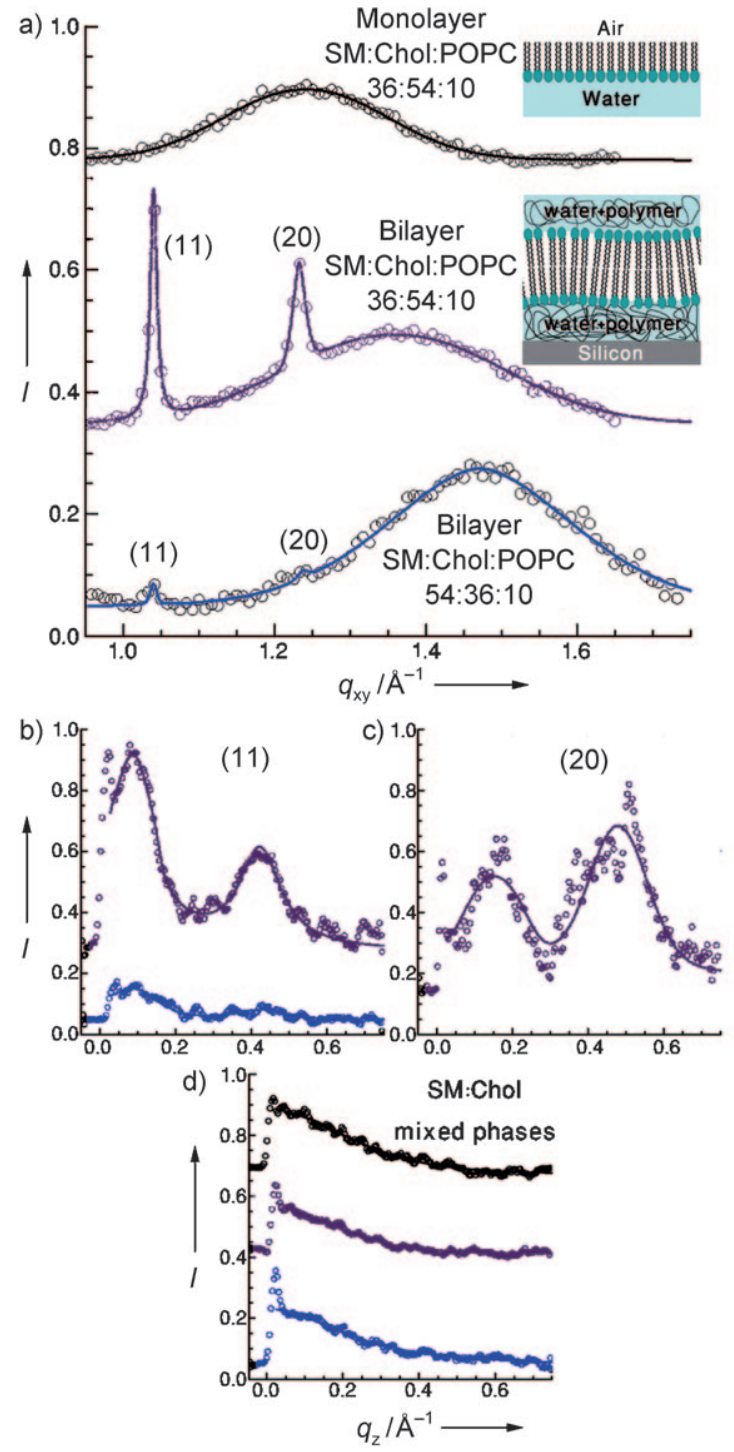

Figure 3. SM:Chol:POPC bilayers and monolayer measured by GIXD. a) Bragg peaks. Top: monolayer on water $\left(6.7^{\circ} \mathrm{C}\right)$. Middle and bottom: bilayers with different compositions. b-d) Bragg rods: b,c) pure Chol (11) and (20) reflections; d) SM:Chol mixed phases. Bragg peaks and rods were fitted with Voight functions (solid lines). The spike at $q_{z} \approx 0.01 \AA^{-1}$ is the Vineyard peak. ${ }^{[14]}$

these peaks are 17-fold weaker than those of the bilayer sample of composition SM:Chol:POPC $=36: 54: 10 \mathrm{~mol} \%$, which indicates, by extrapolation, that pure Chol bilayer crystals begin to form at a composition ratio of SM:Chol:POPC $=56: 34: 10 \mathrm{~mol} \%$. As these experiments were performed at $6.7^{\circ} \mathrm{C}$, at higher temperatures the minimum ratio for pure Chol bilayer formation might be higher.

In conclusion, a method has been presented to obtain diffraction from a single and fully hydrated lipid bilayer. Its proof of concept was demonstrated by obtaining the domain structures of SM:Chol:POPC mixtures. The phase behavior in the lipid bilayer differs greatly from that of the corresponding monolayer. These findings confirm that the structures of bilayers cannot be directly derived by studying their monolayers, and that each case should be studied separately. They

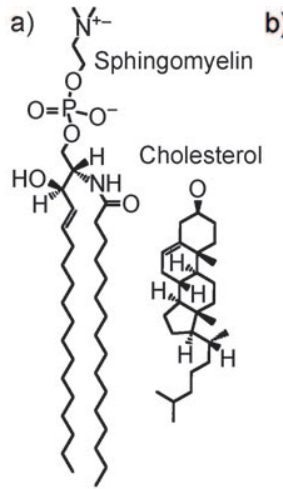

b) c)

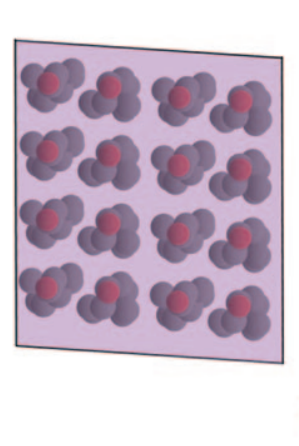

c)

d) Monolayer
SM:Chol:POPC

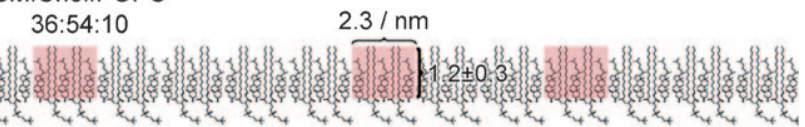

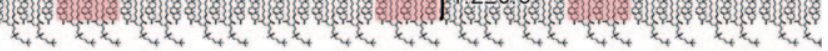

e) Bilayer

SM:Chol:POPC

36:54:10

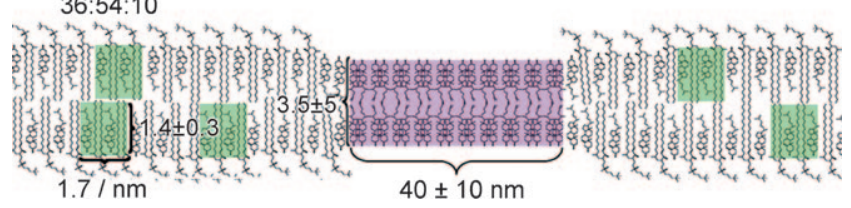

Figure 4. a) SM and Chol chemical formulas. b,c) Unit cell representations of the pure Chol bilayer crystal ${ }^{[6 \mathrm{~d}]}$ and the SM:Chol mixed phase with 2:3 ratio. ${ }^{[6 c]}$ d) Representation of the monolayer SM:Chol:POPC $=36: 54: 10$ mol \%. Red: mixed SM:Chol crystal domains. e) Representation of a bilayer with the same composition. Two crystal domains are formed. Green: mixed SM:Chol crystal domains; purple: pure Chol bilayer-thick crystal. Numbers indicate measured coherence lengths and thicknesses.

also indicate a tendency for phase separation of Chol in the mixed lipid bilayer, to form highly crystalline bilayers by virtue of strong interactions between the Chol exocyclic chain moieties of opposing leaflets. The SM molecules do not phase separate to form crystalline bilayers, perhaps because of the different packing requirements of the head group and chains within and between leaflets. We report the minimum Chol:SM ratio at which pure Chol bilayers are formed, which is not far from the reported ratios in cell plasma membranes. ${ }^{[3]}$ Although the diffraction measurements were performed at $6.7^{\circ} \mathrm{C}$, there is evidence that Chol bilayer-thick crystals may be formed in membranes at body temperature as well. ${ }^{[12]}$ This suggests that such Chol bilayer crystals may form in cells under conditions not far from physiological. These crystals may in turn act as nucleating sites involved in pathological phenomena such as atherosclerosis, cataract, or gall stones. ${ }^{[13]}$

Preliminary results show that this method can also be extended to study samples at $37^{\circ} \mathrm{C}$ (data not shown). The same methodology developed here may be implemented to study lipid domain interactions with proteins. Furthermore, the humidity setup, although designed for bilayer studies, should also be fit to study any $2 \mathrm{D}$ crystals that require 
hydration, thus making it a valuable tool for other biological samples.

Received: July 14, 2009

Published online: October 19, 2009

Keywords: cholesterol · lipid bilayers - monolayers . sphingolipids $\cdot$ X-ray diffraction

[1] S. J. Singer, G. L. Nicolson, Science 1972, 175, 720

[2] a) K. Simons, E. Ikonen, Nature 1997, 387, 569; b) M. F. HanzalBayer, J. F. Hancock, FEBS Lett. 2007, 581, 2098.

[3] G. van Meer, D. R. Voelker, G. W. Feigenson, Nat. Rev. Mol. Cell Biol. 2008, 9, 112.

[4] a) A. H. Futerman, Y. A. Hannun, EMBO Rep. 2004, 5, 777; b) A. H. Futerman, H. Riezman, Trends Cell Biol. 2005, 15, 312

[5] a) J. Als-Nielsen, D. Jacquemain, K. Kjaer, F. Leveiller, M. Lahav, L. Leiserowitz, Phys. Rep. 1994, 246, 251; b) T. R. Jensen, K. Balashev, T. Bjornholm, K. Kjaer, Biochimie 2001, 83, 399.

[6] a) G. Brezesinski, A. Dietrich, B. Struth, C. Bohm, W. G. Bouwman, K. Kjaer, H. Mohwald, Chem. Phys. Lipids 1995, 76, 145; b) I. Kuzmenko, H. Rapaport, K. Kjaer, J. Als-Nielsen, I. Weissbuch, M. Lahav, L. Leiserowitz, Chem. Rev. 2001, 101,
1659; c) L. Scheffer, I. Solomonov, M. J. Weygand, K. Kjaer, L. Leiserowitz, L. Addadi, Biophys. J. 2005, 88, 3381; d) I. Solomonov, M. J. Weygand, K. Kjaer, H. Rapaport, L. Leiserowitz, Biophys. J. 2005, 88, 1809; e) D. Vaknin, M. S. Kelley, Biophys. J. 2000, 79, 2616.

[7] I. Solomonov, K. Kjaer, J. S. Micha, F. Rieutord, G. Fragneto, J. Daillant, L. Leiserowitz, Eur. Phys. J. E 2009, DOI: 10.1140/epje/ i2009-10498-2.

[8] a) T. Salditt, Curr. Opin. Colloid Interface Sci. 2000, 5, 19; b) T. Salditt, G. Brotons, Anal. Bioanal. Chem. 2004, 379, 960.

[9] C. E. Miller, J. Majewski, E. B. Watkins, D. J. Mulder, T. Gog, T. L. Kuhl, Phys. Rev. Lett. 2008, 100, 058103.

[10] J. Y. Wong, J. Majewski, M. Seitz, C. K. Park, J. N. Israelachvili, G. S. Smith, Biophys. J. 1999, 77, 1445.

[11] M. K. Ratajczak, E. Y. Chi, S. L. Frey, K. D. Cao, L. M. Luther, K. Y. C. Lee, J. Majewski, K. Kjaer, Phys. Rev. Lett. 2009, 103, 028103.

[12] G. Kellner-Weibel, P. G. Yancey, W. G. Jerome, T. Walser, R. P. Mason, M. C. Phillips, G. H. Rothblat, Arterioscler. Thromb. Vasc. Biol. 1999, 19, 1891.

[13] T. N. Tulenko, M. Chen, P. E. Mason, R. P. Mason, J. Lipid Res. 1998, 39, 947.

[14] G. H. Vineyard, Phys. Rev. B 1982, 26, 4146. 